\title{
The asymmetry of time and the cellular world. Is immortality possible?
}

\author{
Roberto O. Aquilano ${ }^{1,2}$ \\ ${ }^{1}$ Instituto de Física Rosario (CONICET-UNR), Rosario, Argentina; aquilano@ifir-conicet.gov.ar \\ ${ }^{2}$ Facultad de Ciencias Exactas, Ingeniería y Agrimensura (UNR), Rosario, Argentina
}

Received 21 August 2010; revised 15 October 2010; accepted 10 November 2010.

\begin{abstract}
I analyze the flow of time in this article, both in gross and in microscopic processes, with a well defined arrow of time, but as the amount of energy involved in the microscopic processes is so small, it is more difficult to argue that the entropy increases, and therefore the direction of time becomes confusing and undefined at the molecular level. Therefore, is cell immortality possible?
\end{abstract}

Keywords: Entropy; Time; Cell

\section{INTRODUCTION}

The concept of time is intuitive and easily distinguishable past from present or future. It was not as easy for thinkers. In ancient and found the first human thinking about time. Plato, for example, said that time is the moving image of eternity. Later, Newton described it as an absolute, true and mathematical, which runs smoothly. In the twenties of last century, Einstein came to regard as a mere illusion. These ideas reflect the immense complexity of the time, an issue that has been the subject of reflection for many philosophers and research for many scientists. Scientists are precisely those who now seek to address the fact that science does not provide a clear definition of what is time.

The only fundamental scientific theory that makes a preferred direction for time is of the second law of thermodynamics, which asserts that the entropy of the Universe increases as time flows forward. This explanation provides an orientation, an arrow of time. Our perception of this would, therefore, a direct consequence of the thermodynamic time arrow.

The entropy of any thermodynamically isolated system tends to increase with time and this has to this law a definite orientation. That the entropy of the universe to increase over time is that there is a direction, an arrow of time, a time asymmetry to distinguish past from future, which corresponds with our own perception of time.

This is clear at the macroscopic level, however, on a microscopic scale, since the amount of energy involved in the process is so small, it is more difficult to assert that entropy is increasing, and that therefore time is moving forward (toward the future), rather than backward (toward the past).

In the macroscopic world, how can we explain the obvious time-asymmetry of the universe if the fundamental laws of physic are time-symmetric? Physicists usually answer this question first observing that, if the initial state of the universe would be an equilibrium state, the universe will remain for ever in such state, making impossible to find any time-asymmetry.

The set of irreversible processes that began in an unstable non-equilibrium state constitute a branch system $[1,2]$. That is to say, every one of these processes began in a non-equilibrium state, which state was produced by a previous process of the set.

Once I have understood the origin of the initial unstable state of each irreversible process within the universe it is not difficult to obtain a growing entropy, in any subsystem within the universe. Alternatively, taking into account the enormous amount of information contained in the subsystem we can neglect some part of it $[3,4]$. I can use more refined mathematical tools $[5,6]$. With any one of these tools I can solve this problem.

It remains only one problem: why the universe began in an unstable low-entropy state? If I exclude a miraculous act of creation we have only three scientific answer:

a) The unstable initial state of the universe is a law of nature.

b) This state was produced by a fluctuation.

c) The expansion of the universe (coupled to the nuclear reactions in it) produces a decreasing of the (matter-radiation) entropy gap.

The third solution was sketched by Paul Davies in reference [2], only as a qualitative explanation. The expan- 
sion of the universe is like an external agency (namely: external to the matter-radiation system of the universe) that produces a decreasing of its entropy gap, with respect to de maximal possible entropy, $S_{\max }$ (and therefore an unstable state), not only at $t=0$ but in a long period of the universe evolution. We shall call this difference the entropy gap $\Delta S$, so the actual entropy will be $S_{a c t}=S_{\max }+\Delta S$.

In the microscopic world Feng and Crooks created a method to accurately measure the time asymmetry of the microscopic. In fact have found that, on a microscopic scale and for some intervals, entropy can actually decrease. And that while the general entropy increase on average, each time the experiment does not, that is, time is not always a clear direction. My work aims at understanding the relation between time asymmetry and entropy, which would also be crucial for the development of future molecular and cellular studies.

\section{THE ENTROPY}

We know that the universe isotropic and homogeneous expansion is a reversible process with constant entropy [7]. The matter and the radiation of the universe are in a thermic equilibrium state $\rho^{*}(t)$ at any time t. As the radiation is the only important component, from the thermodynamical point of view, we can chose $\rho^{*}(t)$ as a black-body radiation state.

Let us consider an isotropic and homogeneous model of universe with radius a. From the conservation of the energy-momentum tensor and radiation state equation, we know that $T \sim \mathrm{a}^{-1}$, we can verify that $S=$ const.

The irreversible nature of the universe evolution is not produced by the universe expansion, even if $\rho$ $\rho^{*}(t)$ has a slow time variation. Therefore, the main process that has an irreversible nature after decoupling time is the burning of unstable $\mathrm{H}$ in the stars (that produces $\mathrm{He}$ and, after a chain of nuclear reactions, $\mathrm{Fe}$ ). This nuclear reaction process has certain mean life-time $t_{N R}=\gamma^{-1}$ and phenomenologically we can say the state of the universe, at time $t$, is:

$$
\rho(t)=\rho_{*}(t)+\rho_{1} e^{-\gamma t}+0\left[(\gamma t)^{-1}\right]
$$

where $\rho_{1}$ is certain phenomenological coefficient constant in time, since all the time variation of nuclear reactions is embodied in the exponential law $e^{-\gamma t}$. I can foresee, also on phenomenological grounds, that $\rho_{1}$ must peak strongly around $\omega_{1}$ the characteristic energy of the nuclear process.

All these reasonable phenomenological facts can also be explained theoretically: Eq.1 can be computed with the theory of paper [9] or with rigged Hilbert space theory [5]. In reference [10] it is explicitly proved that $\rho_{1}$ peaks strongly at the energy $\omega_{1}$. The normalization conditions at any time $t$ yields:

$$
\operatorname{tr} \rho(t)=\operatorname{tr} \rho_{*}(t)=1, \ldots \operatorname{tr} \rho_{1}=0
$$

The last equations show that $\rho_{1}$ is not a state but only the coefficients of a correction around the equilibrium state $\rho^{*}(t)$. It is explicitly proved in paper [10], that $\rho_{1}$ has a vanishing trace.

I am now able to compute the entropy gap $\Delta S$ with respect to the equilibrium state $\rho^{*}(t)$ at any time t. It will be the conditional entropy of the state $\rho(t)$ with respect to the equilibrium state $\rho^{*}(t)$ [3]:

$$
\Delta S=-\operatorname{tr}\left[\rho \log \left(\rho_{*}^{-1} \rho\right)\right]
$$

Using now Eq.1, and considering only times $t \gg t_{N R}=\gamma^{-1}$ I can expand the logarithm to obtain:

$$
\Delta S \approx-e^{-\gamma t} \operatorname{tr}\left(\rho_{*}^{-1} \rho_{1}^{2}\right)
$$

where I have used Eq.2. I can now introduce the equilibrium state i for $\omega \gg T$. Then:

$$
\Delta S \approx-Z^{-1} T^{3} e^{-\gamma t} \operatorname{tr}\left(e^{\frac{\omega}{T}} \rho_{1}^{2}\right)
$$

where $\mathrm{e}^{\frac{\omega}{t}}$ is a diagonal matrix with this function as diagonal. But as $\rho_{1}$ is peaked around $\omega_{1}$ we arrive to a final formula for the entropy gap:

$$
\Delta S \approx-C T^{3} e^{-\gamma t} e^{\frac{\omega_{1}}{T}}
$$

where $C$ is a positive constant.

\section{EVOLUTION OF THE ENTROPY GAP $\triangle S$}

I have computed of $\Delta S$ for times larger than decoupling time and therefore, as $a \sim t^{2 / 3}$ and $T \sim a^{-1}$, where $t_{0}$ is the age of the universe and $T_{0}$ the present temperature. Then:

$$
\Delta S \approx-C_{1} e^{-\gamma t} t^{-2} e^{\frac{\omega_{1}}{T_{0}}\left(\frac{t_{0}}{t}\right)^{\frac{2}{3}}}
$$

where $C_{1}$ is a positive constant. The curve $\Delta S(t)$ it has a maximum at $t=t_{c r_{1}}$ and a minimum at $t=t_{c r_{2}}$. Let us compute these critical times. The time derivative of the entropy reads:

$$
\dot{\Delta S} \approx\left[-\gamma-2 t^{-1}+\frac{2}{3} \frac{\omega_{1}}{t_{0} T_{0}}\left(\frac{t_{0}}{t}\right)^{\frac{1}{3}}\right] \Delta S
$$

This equation shows two antagonic effects. The universe expansion effect is embodied in the second and third terms in the square brackets an external agency to the matter-radiation system such that, if we neglect the second term, it tries to increase the entropy gap and, therefore, to take the system away from equilibrium (as we will see the second term is practically negligible). On the other hand, the nuclear reactions embodied in the 
y-term, try to convey the matter-radiation system towards equilibrium. These effects become equal at the critical times $t_{c r}$ such that:

$$
\gamma t_{0}+2 \frac{t_{0}}{t_{c r}}=\frac{2}{3} \frac{\omega_{1}}{T_{0}}\left(\frac{t_{0}}{t_{c r}}\right)^{\frac{1}{3}}
$$

For almost any reasonable numerical values this equation has two positive roots: $t_{c r_{1}} \ll t_{0} \ll t_{c r_{2}}$.

For the first root we can neglect the y $t_{0}$-term and I obtain:

$$
t_{c r_{2}} \approx t_{0}\left(3 \frac{T_{0}}{\omega_{1}}\right)^{\frac{3}{2}}
$$

(this quantity, with minus sign, gives the third unphysical root).

And for the second root I can neglect the $2\left(t_{0} / t_{c r}\right)-t e r m$, and I find:

$$
t_{c r_{2}} \approx t_{0}\left(\frac{2}{3} \frac{\omega_{1}}{T_{0}} \frac{T_{N R}}{T_{0}}\right)^{3}
$$

If I chose appropriate numerical values we can see that it probably produces also a growing order, and therefore the creation of structures like clusters, galaxies and stars [12].

Also I have a growing of entropy, a decreasing order and a spreading of the structures: stars energy is spread in the universe, which ends in a thermic equilibrium [13]. In fact, when $t \rightarrow \infty$ the entropy gap vanishes (see Eq.7) and the universe reaches a thermic equilibrium final state.

Since $10^{4} t_{0} \approx 1.5 \times 10^{14}$ years after the big-bang all the stars will exhaust their fuel [13], so the border between the two periods most likely have this order of magnitude and must also be smaller than this number. This is precisely the result of our calculations.

In the molecular world, we can associate this with the stem cells, seeing that the gap of entropy is close to zero or zero. Feng and Crooks [14] contributed to developing a measure of the time-asymmetry of recent single molecule RNA unfolding experiments.

With the previous calculations but consistent with the body temperatures of animals, we see that the equations are reduced almost naturally to zero entropy, and spread over time, reaching the level of Feng and Crook, producing the possibility that this occurs also mentioned that the cells could become immortal.

\section{CONCLUSIONS}

We see that nature, at the macroscopic level, has a tendency to mess up, which in physics called entropy effect.

\section{Copyright $(2011$ SciRes.}

However, on a microscopic scale, since the amount of energy involved in the processes is very small, it is very difficult to say that entropy is increasing, and therefore time to move forward rather than backward.

Feng and Crooks say have a method to accurately measure time asymmetry at the microscopic level. They found that during some intervals the entropy may decrease. And, although the overall entropy increases at each moment of the experiment does not, then the time has no clear direction and time asymmetry is not secured, but time has a symmetrical (not unlike this past or future). As time progresses in the macroscopic world, it is unclear at the level of a single molecule, and if we associate this phenomenon to stem cells, as these remain unchanged we could say that would be the only natural case of detention of the arrow of time, which could be associated with natural perennial or cell immortality.

\section{ACKNOWLEDGMENTS}

I wish to thank to Jack Szostak of Howard Hughes Medical Institute to fruitful discussions. This work was supported by grant of National University of Rosario (UNR), PID 1ING198.

\section{REFERENCES}

[1] Reichenbach, H. (1956) The direction of time. University of California Press.

[2] Davies, P.C.W. (1994) Stirring up trouble. In: Halliwell, J.J., et al., Ed., Physical Origin of Time Asymmetry, Cambridge University Press, Cambridge.

[3] Mackey, M.C. (1992) Time's arrow: The origin of thermodynamic behavior. Springer Verlag, Berlin.

[4] Zwanzig, R.W. (1966) Statistical mechanics of irreversibility. In: Meijer, P., Ed., Quantum Statistical Mechanics, Gordon and Breach, New York.

[5] Bohm, A. (1986) Quantum mechanics: Foundations and applications. Springer Verlag, Berlin.

[6] Castagnino, M., Gaioli, F. and Sforza, D. (1993) Substantial irreversibility in quantum cosmology. Proceedings of the $V$ International Workshop on Instability and Nonequilibrium Structures, Santiago de Chile, 1993.

[7] Tolman, R.C. (1987) Relativity, thermodynamics, and cosmology. Dover Publications, New York.

[8] Landau, L.D. and Lifshitz, E.M. (1958) Statistical physics. Pergamon Press, Oxford.

[9] Sudarshan, E.C.G., Chiu, C.B., and Gorini, V. (1978) Decaying states as complex energy eigen vectors in generalized quantum mechanics. Physical Review D, 18, 2914-2929. doi:10.1103/PhysRevD.18.2914

[10] Laura, R. and Castagnino, M. (1998) On a minimal irreversible quantum mechanics: The mixed states and de diagonal singularity. Physical Review A, 57, 4140-4142. doi:10.1103/PhysRevA.57.4140

[11] Jones, C. and Forman, W. (1992) Clusters and superclusters of galaxies. In: Fabian, A.C., Ed., NATO ASI Series, Kluwer Academic, Dordrecht, 366, 49-60.

[12] Reeves, H. (1993) The growth of complexity in an ex- 
panding universe. In: Bertola, F. and Curi, U., Ed., The Anthropic Principle, Cambridge University Press, Cambridge.

[13] Dicus, D.A., Letaw, J.R., Teplitz, D.C. and Teplitz, V.L. (1983) The future of the universe. Scientific American,
248, 90-101.

doi:10.1038/scientificamerican0383-90

[14] Feng, E.H. and Crooks, G.E. (2008) Length of time's arrow. Physical Review Letters, 101, 090602-1-090602-4. doi:10.1103/PhysRevLett.101.090602 